\title{
Gestión editorial de publicaciones con referato en línea: proceso de construcción, publicación y administración con soluciones en software libre
}

\author{
Andrés Vuotto, María Carolina Rojas y Gladys Vanesa Fernández \\ Universidad Nacional de Mar del Plata - MDP, Argentina
}

ANÁLISIS

\begin{abstract}
Resumen
Inicialmente se plantean los puntos principales a resolver y desarrollar para la planificación y construcción de una publicación en línea de carácter científico, haciendo hincapié en el proceso y las funciones editoriales, la revisión por pares, la preservación documental, la gestión de acceso, la indexación y visibilidad. En la segunda parte del artículo se expone una propuesta de solución a cada aspecto anteriormente descrito, destacando la labor del profesional de la información y la optimización de tiempos, costos y resultados que ofrece el software libre; a partir de una experiencia concreta con el sistema Open Journal System en el marco del portal de revistas de la Facultad de Humanidades de la Universidad Nacional de Mar del Plata.
\end{abstract}

Palabras clave

Accesibilidad web ; Revistas científicas ; Revistas electrónicas ; Open Journal Systems ; Software libre ; Literatura científica ; Evaluación ; Universidad Nacional de Mar del Plata

Editorial Management serials online: construction process, publication and administration with free software solutions

\begin{abstract}
Initially raised the main points to consider and develop the planning and construction of an online publication of a scientific nature, emphasizing the process and editorial functions, document preservation, access management, indexing and visibility. In the second part of the paper presents a proposed solution to every aspect previously described, highlighting the work of the information professional and optimizing time, cost and results offered free software, from a concrete experience with the system Open Journal System under the journal portal of the Faculty of Humanities at the Universidad Nacional de Mar del Plata.
\end{abstract}

Keywords

Web accessibility ; Scholarly journals ; E-journals ; Open Journal Systems ; Free software ; Scientific literature ; Evaluation ; Universidad Nacional de Mar del Plata

\section{Introdución}

La gestión editorial de publicaciones con referato ha representado en las últimas décadas un espacio en constante cambio, con una marcada orientación hacia la legitimidad en la evaluación del conocimiento registrado y el crecimiento de la visibilidad de la publicación en Internet. La implementación de un sitio web que facilite a los 
lectores el acceso a los textos completos de números vigentes, como anteriores, resulta tan anacrónico como escaso para las reglas que hoy definen el protagonismo y el reconocimiento de las publicaciones científicas.

Desde principios de la década del 90 se ha observado cómo las publicaciones académicas fueron mutando y adaptando sus procesos editoriales al escenario digital; emulando en gran medida aquellos pasos que se cumplían de manera tradicional. De forma paralela al crecimiento que las tecnologías para el desarrollo de la web fueron representando, los sistemas de edición electrónica fueron superando sus obstáculos para el logro de una mayor credibilidad y una usabilidad considerable para cada uno de los roles intervinientes. (1)

\section{Desafíos de los sistemas de edición electrónica}

El proceso editorial de publicaciones académicas plantea diversas complejidades que requieren especial atención. Como son la construcción e implementación de servicios dirigidos directamente a la comunidad de lectores, por ejemplo la administración de suscripciones o accesibilidad libre según corresponda. Pasando por la publicación del número correspondiente, incluyendo la generación de contenidos y sus evaluaciones previas a la aceptación de cada artículo; culminando en la incorporación de dicho número ya editado y publicado al archivo para usos retrospectivos. Priorizando aspectos de almacenamiento, búsqueda, preservación y visibilidad.

Las etapas hasta aquí nombradas (suscripción, accesibilidad y almacenamiento en archivo) son en mayor o menor medida conocidos y conforman los pilares de base para todo sistema de información efectivo.

El nivel mayor de complejidad aparece principalmente en dos puntos hoy considerados claves, como son:

a. El esquema de aprobación de trabajos de los autores como requisito para el reconocimiento de la comunidad científica, y también de organismos evaluadores de la actividad investigadora.

b. Nivel de respuesta del sistema a los estándares de las tecnologías utilizadas, asegurando la accesibilidad de los usuarios y del trabajo de los motores de indexación automatizada.

Para el primer aspecto, la metodología que mejor representa la transparencia y seriedad necesaria es el denominado "doble ciego", donde el archivo a examinar no incluye ningún dato que permita conocer la identidad de sus autores, de esta forma los revisores asignados encuentran un mejor contexto libre de imparcialidades. La característica de doble se completa con la funcionalidad de que los autores tampoco tienen acceso a los nombres de quienes evalúan su artículo.

Para el segundo punto, referido a los estándares, el sistema debe asegurar el uso de tecnologías accesibles y con capacidades para la descripción de contenidos. Sin dudas que el lenguaje XHTML es la herramienta por excelencia para estos casos; aunque también cumple un rol importante el formato de archivos PDF y sus derivados (PDF/A y PDF/X principalmente.) utilizado para la difusión del acceso completo. Una publicación electrónica pura (Fernández Sánchez, Elena; Fernández Morales, Isabel, 2000) (2) debe trabajar ambos formatos, ya que la navegabilidad y uso de las funciones de cada sector del sistema (front-end para los lectores, y back-end para los demás roles intervinientes), como la indexación, se potencian fundamentalmente gracias a las prestaciones del XHTML; mientras que la portabilidad y facilidad de edición es beneficiada por el formato PDF.

En ambos aspectos señalados la correcta intervención de los diferentes actores es de gran importancia para el logro de los resultados. Es una realidad que el sistema debe automatizar y facilitar determinadas funciones, pero la actividad de control constante es una necesidad. La ausencia de revisión de los datos de los autores en los envíos, ya sea en su interior como en las estructuras de propiedades del documento; el olvido de la carga de los metadatos correspondientes a cada artículo y la revisión de expertos previo a su publicación; la falta de entrada de datos en las etiquetas para la descripción de contenidos en el archivo convertido a PDF; la asignación de revisores sin experiencia en la temática del artículo; la publicación del archivo enviado por los autores en lugar de la edición gráfica final; la falta de control en la aceptación de revisiones solicitadas y en el cumplimiento de los plazos establecidos; entre otras tantas situaciones constituyen errores o descuidos frecuentes en los que la responsabilidad no corresponde al sistema sino al factor humano.

\section{Sistemas de publicación de revistas}

Importantes cambios suscitados en el escenario mundial de desarrollo de software, principalmente para funcionamiento online y bajo las leyes del Software Libre, han dado lugar al nacimiento de tecnologías y productos que ofrecen hoy la posibilidad de implementar una editorial virtual que resuelva cada uno de los procesos de edición. 
El entorno por excelencia donde estos sistemas desarrollan todas las actividades es la WWW, en la cual se conjugan varios elementos que interactúan conformando lo que podríamos llamar una "editorial virtual de publicaciones periódicas".

Los lenguajes de etiquetado normalizados por el consorcio W3C (www.w3c.org), como XHTML, CSS, XML y sus derivados; sistemas libres de bases de datos como MySQL, PostgreSQL, etc.; lenguajes de programación y herramientas para montar un servidor generan un marco de trabajo y desarrollo con la performance suficiente como para ofrecer un servicio/producto óptimo para satisfacer en simultaneo grandes comunidades de lectores, autores, editores y revisores.

El número de productos que cubren estas necesidades es suficiente como para poder evaluar y elegir en función de variados criterios; no es menor el dato de que existen otros que se encuentran en desarrollo y en breve también formarán parte de este grupo ya consolidado que año a año ofrece nuevas versiones con significativos avances, beneficiados estos por las comunidades de usuarios y desarrolladores que retroalimentan de manera constante estos sistemas bajo el concepto de software libre.

Nombramos al Open Journal System (3), conocido como OJS, por ser unos de los sistemas pioneros y probablemente el que más adhesiones tiene actualmente en el mundo. Según el registro de julio 2012 realizado por PKP el número de títulos publicado llega a los 12.800

Pero no es el único y nombres como HyperJournal (4), Digital Publishing System (DPubS)(5), ePublishing Toolkit (6), SciXOpen Publishing Services (SOPS) (7) integran la lista.

\section{Selección del sistema adecuado}

Se enumeran las principales condiciones que se consideran como excluyentes a la hora de elegir un sistema de publicación para estos casos:

- El sistema debe operar en un servidor Web

- Su código fuente debe ser abierto, incluyendo cada capa del back-end o de las partes que procesan la información enviada por el usuario para ofrecer una respuesta según la interacción planteada por este (capa lógica o de negocio, y capa de datos), de esta forma será posible acceder a cada parte del sistema y modificarlo según necesidades y/o conflictos presentados.

- $\quad$ El sector front-end, o interfaces que interactúan con el usuario, debe ser acorde a la usabilidad que requiere el producto, ofreciendo una arquitectura que reconozca los diferentes niveles de información, y reconociendo cada uno de los roles que tomarán decisiones dentro del proceso de edición, contando también para este caso a los lectores reales y potenciales.

- El motor de búsqueda debe ofrecer una interface completa y un funcionamiento óptimo ya que la búsqueda de números anteriores o de tipo temática dentro del archivo de la publicación es una tarea frecuente y muy evaluada por el público al cual se destinan estos servicios.

- El sistema debe permitir generar backup de forma sencilla, principalmente de las bases de datos y sus registros, como también de los artículos de las publicaciones y de la información de los usuarios registrados.

- Debe cumplir con los estándares establecidos para los productos Web y para la recuperación de información de objetos digitales, asegurando una mejor y correcta indexación y posicionamiento de la publicación, ya sea con respecto a los buscadores como también con los sitios pares. En este aspecto la ausencia de plugins del tipo RIA (Rich Internet Applications) es muy importante, ya que los mismos no suelen cumplir con los estándares y la entrega de contenidos que realizan suele ser problemática tanto para su búsqueda y recuperación, como para la correcta visualización en los diversos browsers. Los casos de Adobe Flash y Microsoft Silverlight son los más característicos.

- El módulo o sección del sistema destinado al proceso de referato debe ser tan claro como completo. En este caso la posibilidad de evaluación por parte de expertos de tipo doble ciego, y el seguimiento de la evolución del proceso de corrección y mejora del artículo 
antes de su edición, es un factor estratégico y con gran impacto en la calidad del producto final. Los principales actores de esta etapa, como autores y revisores, deben contar con un panel que gestione y deje asentado cada paso que va cumpliendo el artículo desde que ingresa al comité evaluador y hasta que culmina en la publicación propiamente dicha.

- Su visualización y navegación debe funcionar correctamente en los principales navegadores web, como también en las más utilizadas plataformas operativas.

- La comunidad de usuarios (publicaciones que hacen uso del sistema y/o programadores) deben presentar una actividad vigente y responsable, asegurando de esta forma un soporte técnico o especialistas a los cuales recurrir ante consultas o posibles problemáticas.

La organización PKP, destinada a mejorar la calidad académica y pública de la investigación, presenta un gráfico que si bien está acotado a la actividad del software editorial que dicha organización desarrolla y mantiene (como es el caso del Open Journal System); el mismo puede aplicarse a un caso generalizado de sistema de edición. Este esquema muestra los circuitos de información que una "editorial virtual" para publicaciones en línea debe cumplimentar, y quiénes son los actores que operan en dicho circuito (figura 1).

Figura 1 - Proceso Editorial y de Publicación de OJS

\section{Autor \\ Editor y Editor de Sección}

Suscripción
El autor puede subir el archivo al sitio
Web del diario, y entrar los metadatos
por una indexación con una Interfaz
Orientada a Objetos

Revisión de la suscripción El autor puede chequear el proceso, ver las archivos, las revisiones, hace correcciones y reinscribirse a petición del editor. a) Comprobar la suscripción. b) Realizar revisión paritaria.
1. Pasos de Suscripción Editor asigna la suscripción al Editior ode Sección el cual lo comprobara a través del proceso editiorial.
Administrador del Diario, Revisor, Editor de Copia, Editor de Diseño, y Lector de Prueba

Administración del Diario La configuración y establecimiento de un diario incluye editores, revisores editores de capia, y lectares de prueba.

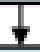

Selecionar revisores El editor invita a revisores con grar niteres en el tema que forman parte de c) Llegar a una decisión editorial.

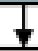

Revisión Acesorada

Los revisores dan a conocer sus resultados y recomendaciones (los cuales pueden ser evaluadas por e editar)

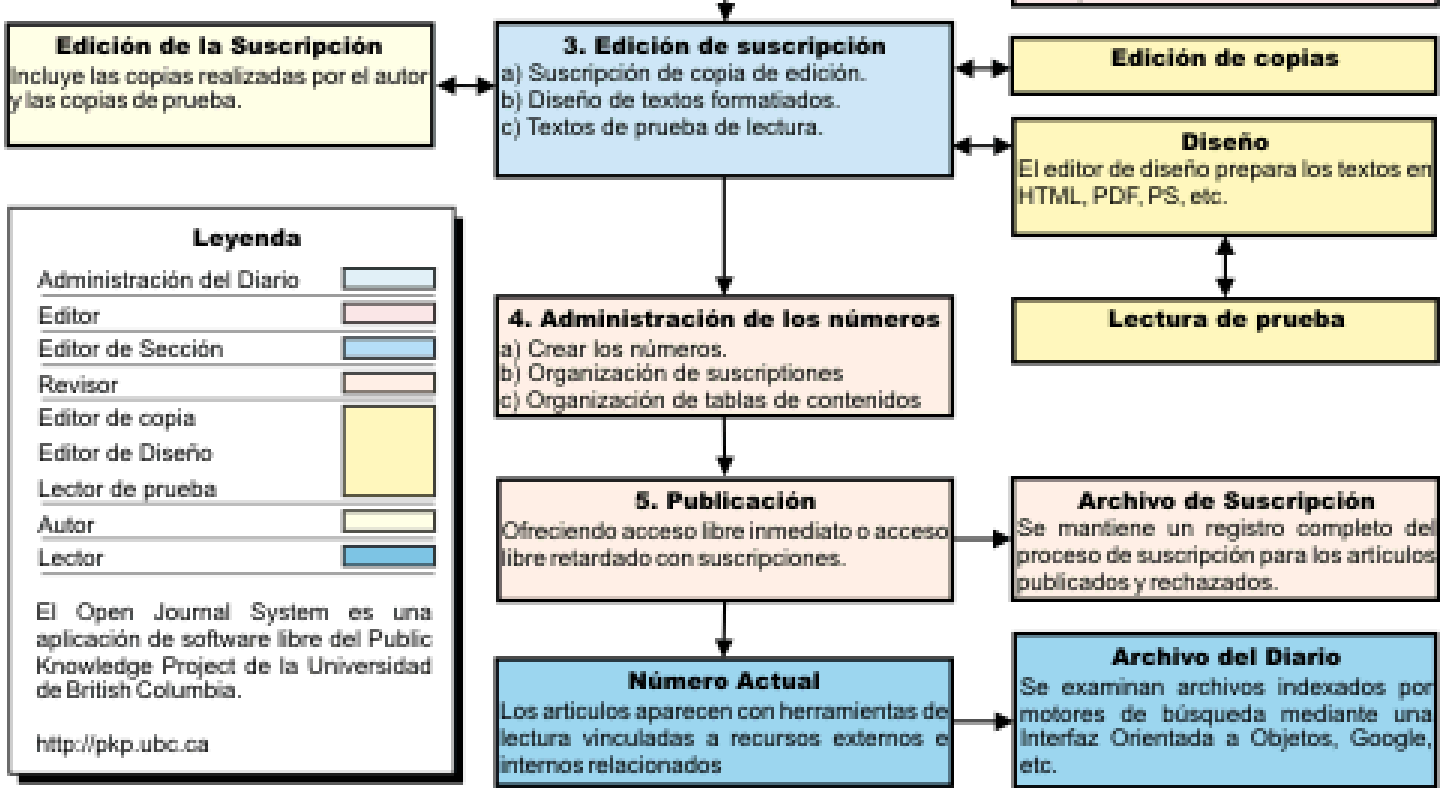

Fuente: https://pkp.ubc.ca/ 


\section{El caso del Sistema OJS}

Un sistema que cumple con buenos resultados los requisitos ya planteados es el desarrollo de la organización Public Knowledge Project (http://pkp.sfu.ca) conformada por Facultad de Educación de la Universidad de la Columbia Británica, la Biblioteca de la Universidad Simon Fraser, de la Escuela de Educación de la Universidad de Stanford, y el Centro Canadiense de Estudios de la Industria Editorial en la Universidad Simon Fraser.

El software Open Journal Systems, conocido como OJS, consiste en un programa de código abierto disponible para cualquier publicación del mundo y cuyo principal objetivo es acompañar cada etapa del proceso de publicación con referato. Algunos aspectos, al margen de los ya enumerados como excluyentes, que hacen del OJS un sistema óptimo para considerar en la planificación e implementación de una publicación con referato en línea son los siguientes:

a) Oficialmente hay en el mundo más de 12000 publicaciones que lo adoptan, que junto con el respaldo de la PKP, lo hacen un producto en constante evolución y procesos de mejora. $Y$ considerando un escenario con reglas tan cambiantes como es el de la WWW, no es un detalle menor; ya que la incorporación constante de las modificaciones de los viejos estándares como de los nuevos, y el uso de las potencialidades de las nuevas versiones de lenguajes de programación y sistemas de bases de datos, influyen directamente en las prestaciones y la usabilidad del software.

b) Es un sistema multilingüe, con soporte para más de 30 lenguas. Lo que permite trabajar con interfaces en diferentes lenguas según la necesidad, como también ofrecer la publicación a los lectores en diferentes lenguajes con solo pocos pasos de configuración.

c) Está completamente desarrollado con tecnologías libres, como son el caso de PHP, MySQL o PostgreSQL, y funciona con buenos rendimientos en el servidor Apache sin sobrecargar el mismo, lo cual no exige grandes inversiones en hardware.

d) Ofrece soporte para varias publicaciones, lo que posibilita la administración de más de una revista dentro de la plataforma, cada una con sus paneles independientes según las etapas del proceso de edición y de referato de cada una.

\section{La experiencia a partir de las implementaciones y migraciones realizadas}

La Revista Electrónica de Historia de la Comunicación, bajo la dirección de la Lic. Aurora Chiriello, nació como una necesidad de dar a conocer las investigaciones sobre historia de la comunicación que se realizan en el ámbito del sudeste de la provincia de Buenos Aires.

Constituye una publicación en formato digital desde su primer minuto de vida. Pero por aquellos años (1999) la posibilidad de un sitio web no era algo corriente como por estos tiempos, por lo que en sus inicios se presentaba en un formato compilado y distribuido en soportes portátiles como los discos de 3 1⁄2.

En su "segunda etapa", año 2003, incorpora el servicio Web (http://www.rehisco.com.ar) para acceder a los textos completos de los números ofrecidos con periodicidad anual, en un formato estático.

En su "tercera etapa", con desarrollo en curso, el objetivo es incorporar la revista (números anteriores y venideros) a los formatos y estándares internacionales a partir de la migración a una plataforma de publicación virtual con referato; manteniendo como plazo máximo diciembre de 2010.

Como primer paso se estipuló desarrollar un análisis de requisitos a cumplir por el sistema elegido, sin que esto significase un alto costo económico y de capacitación. Ya que el equipo a cargo cuenta con experiencia en 
desarrollos similares con tecnologías libres, y capitalizar la misma en una nueva experiencia forma parte de los objetivos permanentes del trabajo.

El sistema OJS reúne dichas condiciones, y luego de la aprobación del mismo como la plataforma tecnológica para la nueva edición de La Revista, se continuó con el diseño y publicación de la misma en un servidor de la Facultad de Humanidades (http://fh.mdp.edu.ar/revistas), que se encuentra disponible para la publicación de revistas de la institución y teniendo en cuenta que ya había sido publicado el primer número de la Revista de Educación (http://fh.mdp.edu.ar/revistas/index.php/r educ).

Al tratarse de una revista con varios números publicados, se procedió a diseñar un plan de conversión de los números anteriores de la revista a texto completo y el rediseño de esta en función de las necesidades de la nueva plataforma, siguiendo los siguientes pasos:

a) Recopilación de los números publicados años anteriores en diskette y en la Web.

b) Diseño de maquetación en OpenWriter (perteneciente al paquete OpenOffice) de los artículos para publicar posteriormente en formato pdf.

c) Generación de archivos pdf a partir de la combinación de los artículos con la plantilla diseñada.

d) Creación de la Revista de Historia de la Comunicación en la plataforma OJS instalada en la Facultad de Humanidades.

e) Creación de cada uno de los números publicados e integración de las descripciones de metadatos y archivos correspondientes a cada número.

f) Publicación y disposición al público en el servidor.

Se quiere prestar especial atención en que las herramientas utilizadas para la realización de todos los procesos son de software libre.

\section{Conclusiones}

Como se nombró a lo largo de este artículo, el respaldo tecnológico y el cumplimiento de pasos editoriales y de evaluación ciega son claves en una correcta gestión de publicaciones electrónicas de tipo académicas. El primero responde en términos generales a la accesibilidad y a la gestión virtual de cada proceso, mientras que el segundo refiere a particularidades que conciernen al rigor científico de la publicación y tienen un alto impacto en la evaluación que esta reciba de la comunidad científica.

Los estándares y las herramientas libres tienen una influencia directa en el crecimiento del servicio, ya que la Web y las organizaciones que la reglamentan (W3C; WHATWG; y demás empresas y fundaciones como pueden ser Apple, Opera, Fundación Mozilla, entre otras) cada vez ponen mayor énfasis en estos aspectos, contrarrestando la presencia de muchos conocidos plugins que, si bien en muchos casos han ofrecido algún que otro embellecimiento multimedia en un sitio web, en su mayoría atentan sustancialmente contra la navegabilidad, arquitectura de la información, indización, búsqueda y recuperación, minimizando el principal objetivo de la Web como es la accesibilidad e intercambio de información en pro de un crecimiento de las posibilidades de aprender, registrar y generar conocimiento.

Diversos factores que configuran el escenario actual, como también la reglas de aplicación de indicadores para la evaluación de la producción científica y de sus publicaciones, plantean la necesidad de administrar el proceso 
editorial por medio de sistemas gestores que configuran un marco propicio para responder con buenos rendimientos a las exigencias detalladas.

En función de las experiencias abordadas en nuestra unidad académica con varias publicaciones, encontramos al profesional de la información como un actor fundamental para tareas de tipo tecnológicas, de gestión, administración y asesoramiento a comités editoriales y sectores directivos de las propias publicaciones. No sólo por conocer las técnicas y procesos necesarios para el almacenamiento y recuperación de la información, sino también por dominar cada vez con mayor profundidad dos tópicos que hemos abordado en detalle. Como son la evaluación de la producción de las investigaciones registradas en publicaciones periódicas y el desarrollo de documentos electrónicos a partir de estándares vigentes asegurando su recuperación, indización automatizada, interoperabilidad con sistemas de harvester y perdurabilidad en el tiempo.

\section{Notas}

1. Se pueden nombrar los autores, editores de sección, editores generales, gestor de revista, revisores, director, administrador de sistemas, entre otros.

2. Se denominan revistas electrónicas puras a aquellas que nacieron exclusivamente para la web, y todo su proceso de edición se llevan a cabo por medios digitales.

3. Desarrollado por Public Knowledge Project (http://pkp.sfu.ca/)

4. Más información en http://www.hjournal.org/

5. Cornell University Library (http://www.cornell.edu/)

6. Living Reviews Publishing Toolkit (https://dev.livingreviews.org/projects/epubtk/)

7. SCIx (http://www.scix.net/)

\section{Bibliografía}

Fernández Sánchez, E.; Fernández Morales, I (2000). Consideraciones sobre la edición electrónica de revistas en internet. En: El profesional de la información, marzo, v. 9, n. 3, pp. 4-12. Disponible en:

http://www.elprofesionaldelainformacion.com/contenidos/2000/marzo/2.pdf

Jiménez-Hidalgo, S.; Giménez-Toledo, E.; Salvador-Bruna, J. (2008). Los sistemas de gestión editorial como medio de mejora de la calidad y la visibilidad de las revistas científicas. En: El profesional de la información, 2008, mayojunio, v. 17, n. 3, pp. 281-291. Disponible en:

http://www.elprofesionaldelainformacion.com/contenidos/2008/mayo/04.pdf

Kopak, R.; Chiang, C. (2009). An interactive reading environment for online scholarly journals: The Open Journal Systems reading tools. En: OCLC Systems \& Services, v. 25 n. 2, pp.114-124. Disponible en:

http://dx.doi.org/10.1108/10650750910961910

Maquilón Sánchez, J.; Lillo Hidalgo, V.; Mirete Ruiz, A. (2011). La edición electrónica y la gestión de trabajos académicos con Open Conference Systems (OCS). En: Revista electrónica interuniversitaria de formación del profesorado, v. 14, n. 1, pp. 325-344. Disponible en: http://aufop.com/aufop/uploaded files/articulos/1302732163.pdf 
Sánchez Caballero, Matías (2010). Software libre y accesibilidad. En: No Solo Usabilidad, № 9, 2010.

$<$ http://nosolousabilidad.com>. ISSN 1886-8592

Sánchez Tarragó, N.; Díaz Alvarez, Y (2005). El sector editorial contemporáneo y las competencias profesionales.

En: Acimed, septiembre - octubre, v. 13 (5).

Suber, P (2004). Promoting open access in the humanities, Annual Meeting of the American Philological Association. En: Annual Meeting of the American Philological Association, pp 231-246. Disponible en:

http://dash.harvard.edu/bitstream/handle/1/4729720/suber promoting.htm? sequence=1

Voces, R.; Codina, L (2008). La accesibilidad potencial y real del formato pdf: Análisis de diarios digitales españoles. En: El profesional de la información, marzo-abril, v. 17, n. 2, pp. 205-212. Disponible en:

http://www.elprofesionaldelainformacion.com/contenidos/2008/marzo/11.pdf

Willinsky , J (2005). Open Journal Systems: An example of open source software for journal management and publishing. En: Library Hi Tech, v. 23 n. 4, pp.504-519. Disponible en: http://dx.doi.org/10.1108/07378830510636300

\section{Datos de los autores}

Andrés Vuotto

Jefe de Trabajos Prácticos con dedicación exclusiva a la docencia e investigación, perteneciente al área de Procesamiento de la Información, Departamento de Documentación, Universidad Nacional de Mar del Plata.

avuotto@gmail.com

María Carolina Rojas

Profesor Adjunto con dedicación exclusiva a la docencia e investigación, perteneciente al área de Procesamiento de la Información, Departamento de Documentación, Universidad Nacional de Mar del Plata. metacaro@gmail.com

Gladys Vanesa Fernández

Ayudante de Primera con dedicación exclusiva a la docencia e investigación, perteneciente al área de Procesamiento de la Información, Departamento de Documentación, Universidad Nacional de Mar del Plata.

gvfernan07@gmail.com

$\begin{array}{ll}\text { Recibido - Received } & : 2013-08-25 \\ \text { Aceptado - Accepted } & : 2013-09-27\end{array}$




\section{(c) EY $_{\text {EY }}$ New articles in this journal are licensed under a Creative Commons Attribution 3.0} United States License.

\section{ULIS D-Surk}

This journal is published by the University Library System of the University of Pittsburgh as part of its D-Scribe Digital Publishing Program and is cosponsored by the University of Pittsburgh Press. 